\title{
Identification of seeds based
} on molecular markers and secondary metabolites in Senna obtusifolia and Senna occidentalis

\author{
Renjun Mao ${ }^{1}$, Pengguo Xia², Zhigui He ${ }^{1}$, Yan Liư ${ }^{3}$, Fenghua Liư ${ }^{4}$, Hongguang Zhao ${ }^{4}$, Ruilian Han ${ }^{1}$ \\ and Zongsuo Liang ${ }^{1,2^{*}}$
}

\begin{abstract}
Background: Senna obtusifolia and Senna occidentalis (Leguminosae), whose seeds have similar appearance and chemical constituents, are easily confused in using their seeds. To elucidate the similarities and differences between S. obtusifolia seeds and S. occidentalis seeds, three molecular markers and high performance liquid chromatography (HPLC) were employed to evaluate the seeds characteristics of these two medicinal herbs.

Results: The results showed that selected 3 ISSR and 7 SCOT primers could distinguish S. obtusifolia seeds from S. occidentalis seeds based on the specific band and UPGMA dendrogram. ITS2 sequence indicated that the intra-specific similarity of 20 S. obtusifolia and 16 S. occidentalis was 99.79 and 100.0\%, respectively, while the inter-specific similarity between S. obtusifolia and S. occidentalis was $89.58 \%$. Although phylogenetic analysis revealed that these two species had a close relationship, they were assigned to different branches. HPLC fingerprint results showed that seeds of $S$. obtusifolia and S. occidentalis shared some secondary metabolites, but aurantio-obtusin was not detected in S. occidentalis seeds which could differentiate $S$. obtusifolia seeds from $S$. occidentalis seeds.

Conclusions: The present study not only compared the seeds characters of S. obtusifolia and S. occidentalis from molecular and secondary metabolites levels, but also provided a convenient method to identify S. obtusifolia seeds and S. occidentalis seeds effectively.
\end{abstract}

Keywords: S. obtusifolia seeds, S. occidentalis seeds, Molecular marker, ITS2 Sequence, HPLC fingerprint

\section{Background}

Senna obtusifolia L. (Leguminosae) whose seeds known as Juemingzi in China, is a famous traditional Chinese medicine and has been regarded as a food and drug dual-purpose material by China Food and Drug Administration (CFDA). S. obtusifolia seeds owned various pharmaceutical properties such as eyesight improvement (Yang et al. 2012), blood lipid regulation, hypertension regulation (Li and Guo 2002) and hepatoprotective effect

\footnotetext{
*Correspondence: liangzs@ms.iswc.ac.cn

1 State and Local Joint Research Center of TCM Fingerprint and NP Library, College of Life Sciences, Northwest A\&F University, Yangling 712100, Shaanxi, China

Full list of author information is available at the end of the article
}

(Kim et al. 2009). These properties make S. obtusifolia seeds very popular in China and some other Asian countries. Since the wide applications in pharmaceutical and healthcare industries, the demand of S. obtusifolia seeds increased rapidly, and the proportion of its adulterant increased at the same time. Among the adulterants, the most common and indistinguishable one is the seeds of Senna occidentalis L., which is an ayurvedic medicinal herb also belong to genus Senna Leguminosae family. Several investigations had demonstrated that S. occidentalis have antibacterial (Jafri et al. 1999; Saganuwan and Gulumbe 2006), antimutagenic (Jafri et al. 1999), antiplasmodial (Tona et al. 2004) and hepatoprotective activities (Yadav et al. 2010). 
Unfortunately, as the non-staple traditional medicine, the research level of S. obtusifolia and S. occidentalis is limited. The published papers about these two species mainly focus on their pharmacological characteristics (Drever et al. 2008; Ju et al. 2010) and chemical structures (Sob et al. 2008; Li et al. 2010). The authenticity of S. obtusifolia seeds is still identified by some traditional methods such as appearance characters or microstructures. The traditional identification methods are largely dependent on subjective experience and feelings, which easily bring confusion in identifying of $S$. obtusifolia seeds and $S$. occidentalis seeds. Even more alarming is that improper use of $S$. occidentalis seeds will cause serious symptoms (Panigrahi et al. 2014; Teles et al. 2015). However, there has no systematic research from the molecular and secondary metabolite levels to identify S. obtusifolia seeds and S. occidentalis seeds. Therefore, it is imperative to elucidate the similarities and differences between S. obtusifolia seeds and S. occidentalis seeds, establishing a suitable method to distinguish them.

In the present study, $20 \mathrm{~S}$. obtusifolia samples and 16 S. occidentalis samples were collected mainly from China. ISSR and SCoT markers, ITS2 sequence and HPLC fingerprint were used to compare the differences between S. obtusifolia seeds and S. occidentalis seeds, and thereby providing an efficient method to identify them.

\section{Methods}

Plant materials

20 S. obtusifolia samples and 16 S. occidentalis samples were collected during 2012-2015. Detailed information of each sample had been list in Table 1 . All samples were identified by Professor Yuejin Zhang (College of Life Sciences, Northwest A\&F University) and voucher specimens (Specimens number see Table 1) were deposited in the State and Local Joint Research Center of TCM Fingerprint and Natural Product Library, Northwest A\&F University.

\section{DNA extraction}

30 sterilized seeds of each sample were placed in illumination incubator to germinate under $28^{\circ} \mathrm{C}, 2500 \mathrm{~lx}(8 \mathrm{~h} /$ day) and $75 \%$ relative humidity conditions for 5 days. Young and healthy leaves from ten individuals of each sample were randomly collected and mixed for genomic DNA extraction. Total genomic DNA was extracted by using New NuClean Plant Genomic DNA Kit (CWBIO, China) according to the manufacturer's instructions. The quality and concentration of DNA was examined by $1 \%$ agarose gel electrophoresis and spectrophotometer analysis (NanoDrop 1000, Thermo Scientific).

\section{PCR programs and ITS2 sequencing}

ISSR and SCoT PCR reactions were conducted in $20 \mu \mathrm{L}$ volume containing $10 \mu \mathrm{L} 2 \times$ Taq Mix (CWBIO, China),

Table 1 Detailed information of 36 samples used in the present study

\begin{tabular}{|c|c|c|c|c|c|c|c|c|c|}
\hline No. & Name & Haplotypes & GenBank accessions & Voucher no. & No. & Name & Haplotypes & GenBank accessions & Voucher no. \\
\hline 1 & $\mathrm{HN}-\mathrm{DZ}-12^{\mathrm{a}}$ & A1 & MF061316 & TCMNP-1241 & 19 & JS-TZ-14 & $\mathrm{A} 2$ & MF061317 & TCMNP-1481 \\
\hline 2 & HN-ZMD-12 & A3 & MF085547 & TCMNP-1242 & 20 & $\mathrm{ZJ}-\mathrm{HZ}-15$ & A3 & MF085555 & TCMNP-1571 \\
\hline 3 & HN-ZMD-13 & A3 & MF085550 & TCMNP-1341 & 21 & SX-YL-O13 & B1 & MF085530 & TCMNP-0301 \\
\hline 4 & $\mathrm{HN}-\mathrm{ZMD}-14^{\mathrm{a}}$ & A3 & MF085548 & TCMNP-1441 & 22 & SX-YL-O14 & B1 & MF085531 & TCMNP-0401 \\
\hline 5 & $S X-Y L-13$ & A1 & MF067418 & TCMNP-1301 & 23 & SX-YL-O15 & B1 & MF085532 & TCMNP-0501 \\
\hline 6 & $S X-Y L-14$ & $\mathrm{~A} 1$ & MF067419 & TCMNP-1401 & 24 & SX-SL-O14 & B1 & MF085533 & TCMNP-0402 \\
\hline 7 & $S X-Y L-15$ & A1 & MF067420 & TCMNP-1501 & 25 & SX-SL-015 & B1 & MF085534 & TCMNP-0502 \\
\hline 8 & SX-SL-15 & A3 & MF085549 & TCMNP-1502 & 26 & SX-LN-O14 & B1 & MF085535 & TCMNP-0403 \\
\hline 9 & SX-WN-14 & A1 & MF067421 & TCMNP-1402 & 27 & HN-DZ-O14 & B1 & MF085536 & TCMNP-0441 \\
\hline 10 & SX-LN-14 & A1 & MF067422 & TCMNP-1503 & 28 & YN-KM-O14 & $\mathrm{B} 1$ & MF085537 & TCMNP-0461 \\
\hline 11 & SX-LN-15 & A1 & MF067423 & TCMNP-1403 & 29 & JS-YZ-O14 & B1 & MF085538 & TCMNP-0481 \\
\hline 12 & Vietnam-14 & A1 & MF067424 & TCMNP-14F1 & 30 & JS-HA-O14 & $\mathrm{B} 1$ & MF085539 & TCMNP-0482 \\
\hline 13 & Myanmar-14 & $\mathrm{A} 2$ & MF067425 & TCMNP-14F2 & 31 & GX-NN-O15 & B1 & MF085540 & TCMNP-0551 \\
\hline 14 & SC-CD-14 & A3 & MF061318 & TCMNP-1491 & 32 & GX-BS-015 & B1 & MF085541 & TCMNP-0553 \\
\hline 15 & SD-HZ-15 & A3 & MF085551 & TCMNP-1531 & 33 & GX-YL-O15 & $\mathrm{B} 1$ & MF085542 & TCMNP-0552 \\
\hline 16 & HB-AG-15 & A3 & MF085552 & TCMNP-1521 & 34 & GD-MM-O14 & B1 & MF085543 & TCMNP-0431 \\
\hline 17 & AH-BZ-14 & A3 & MF085553 & TCMNP-1461 & 35 & GD-GZ-014 & B1 & MF085544 & TCMNP-0432 \\
\hline 18 & GX-YL-15 & A3 & MF085554 & TCMNP-1551 & 36 & JX-PX-O14 & B1 & MF085545 & TCMNP-0493 \\
\hline
\end{tabular}

HN-ZMD-14 indicated that the sample was collected from Zhumadian city, Henan province in 2014

a Indicated these samples were collected from wild field and the other samples were collected from the cultivars fields or experimental fields 
$8 \mu \mathrm{L} \mathrm{ddH}_{2} \mathrm{O}, 1 \mu \mathrm{L}$ primer $(1 \mathrm{mM}), 1 \mu \mathrm{L}$ template DNA $(20 \mathrm{ng} / \mu \mathrm{L}) .100 \mathrm{ISSR}$ and $85 \mathrm{SCoT}$ primers were based on University of British Columbia and Collard and Mackill (Collard and Mackill 2009), respectively. All the primers were synthesized by Sangon Biological Engineering Technology \& Services Company (Shanghai, China). ISSR-PCR reaction started at $94{ }^{\circ} \mathrm{C}$ for $5 \mathrm{~min}$, followed by 35 cycles of $94{ }^{\circ} \mathrm{C}$ for $45 \mathrm{~s}, 55^{\circ} \mathrm{C}$ for $40 \mathrm{~s}$ and $72{ }^{\circ} \mathrm{C}$ for $1.5 \mathrm{~min}$, the final extension step at $72{ }^{\circ} \mathrm{C}$ was held for $7 \mathrm{~min}$. SCoT-PCR reaction started at $94{ }^{\circ} \mathrm{C}$ for $5 \mathrm{~min}$, followed by 35 cycles of $30 \mathrm{~s}$ at $94{ }^{\circ} \mathrm{C}, 1 \mathrm{~min}$ at $50{ }^{\circ} \mathrm{C}$ and then $1.5 \mathrm{~min}$ at $72{ }^{\circ} \mathrm{C}$, with the extension at $72{ }^{\circ} \mathrm{C}$ for $7 \mathrm{~min}$.

The PCR reaction for ITS2 was conducted in $50 \mu \mathrm{L}$ volume containing $25 \mu \mathrm{L} 2 \times$ Taq Mix, $19 \mu \mathrm{L}$ $\mathrm{ddH}_{2} \mathrm{O}, 2 \mu \mathrm{L}$ primer forward $(1 \mathrm{mM}), 5^{\prime}$-ATGCGATACTTGGTGTGAAT- $3^{\prime}, 2 \mu \mathrm{L}$ primer reverse $(1 \mathrm{mM})$, $5^{\prime}$-GACGCTTCTCCAGACTACAAT- $3^{\prime}$ and $2 \mu \mathrm{L}$ template DNA $(20 \mathrm{ng} / \mu \mathrm{L})$. ITS2 PCR reaction started at $94{ }^{\circ} \mathrm{C}$ for $5 \mathrm{~min}$, followed by 40 cycles of $94{ }^{\circ} \mathrm{C}$ for $30 \mathrm{~s}, 56^{\circ} \mathrm{C}$ for $30 \mathrm{~s}$ and $72{ }^{\circ} \mathrm{C}$ for $45 \mathrm{~s}$, the final extension step at $72{ }^{\circ} \mathrm{C}$ was held for $10 \mathrm{~min}$ (Chen et al. 2010). PCR reactions were performed in a Veriti 96 Thermal Cycler (Applied Biosystems, USA). PCR productions were visualized by agarose gel electrophoresis and sequenced by AuGCT Technology \& Services Company (Beijing, China).

\section{Determination of secondary metabolites content}

The secondary metabolites were determined by HPLC system (Waters, Milford, USA) equipped with a 1525 binary pump and 2487 Dual $\lambda$ detector. The column was Waters Sunfire $C_{18}$ reserved-phase column $(5 \mu \mathrm{m}$, $250 \mathrm{~mm} \times 4.6 \mathrm{~mm}$ ). The secondary metabolites extraction and chromatography condition were according to the method described in Chinese Pharmacopoeia (Chinese Pharmacopoeia Commission 2015). Standard references of aurantio-obtusin (111900-201504), chrysophanol (110796-201520), physcion (110758-200611), emodin (110756-200110) and aloe-emodin (110795201308) were purchased from the National Institute for the Control of Pharmaceutical and Biological Products (Beijing, China). Every sample was detected with three technical replicates to reduce the error rate.

\section{Data analysis}

Every PCR reaction repeated twice and only unambiguous and repeatable bands were taken into account. NTSYS-pc 2.10e was employed to calculate the similarity coefficient (Rohlf 1993). The dendrograms were generated from similarity matrix data by cluster analysis using unweighted pair group method for arithmetic mean (UPGMA). The raw sequences were assembled by Codon Code Aligner V 4.0.3 (Codon Code Co., USA) based on the Hidden Markov model (HMMer) annotation method (Tamura et al. 2013). The sequence divergence and genetic distance were performed with pairwise calculations under Kimura 2-parameter model by using MEGA 6.06 software. Totally 54 samples, including 20 S. obtusifolia, 16 S. occidentalis, 15 closely related samples and three Glycine max samples, i.e., Senna italica CIMAP-C026 (Genbank Accession Number: KY492294), Senna italica CIMAP-C033 (KY492295), Senna tora CIMAP-C044 (KY492308), Senna alexandrina CIMAP-C032 (KY492292), Senna alexandrina CIMAP-C039 (KY492293), Senna uniflora CIMAP-C043 (KY492305), Senna italica (JQ301844), Senna spectabilis CIMAP-C027 (KY492297), Senna spectabilis CIMAPC034 (KY492298), Senna spectabilis CIMAP-C041 (KY492299), Senna tora CIMAP-C037 (KY492307), Senna tora CIMAP-C044 (KY492308), Senna auriculata CIMAP-C028 (KY492300), Senna auriculata CIMAPC035 (KY492301), Senna auriculata CIMAP-C042 (KY492302), Glycine max SL2 (KP793718), Glycine max 001 (AJ009787) and Glycine max Williams (KP793718), were used to construct phylogenetic tree by using Neighbor jointing (NJ) and Maximum likelihood (ML) methods, respectively.

\section{Results \\ Discrimination ability and polymorphism of ISSR and SCOT markers}

Among 100 ISSR primers, 3 primers (Table 2) could generate specific band that distinguish S. obtusifolia from S. occidentalis. The primer UBC80 generated a specific band about 860 bp in $S$. occidentalis samples which did not observe in S. obtusifolia samples (Fig. 1). The primers UBC81 and UBC85 yielded the specific bands, which could distinguish $S$. obtusifolia from S. occidentalis (Additional file 1: Figure S1). 3 ISSR primers totally yielded 70 bands among 20 S. obtusifolia samples, 51 of which were polymorphic bands with $72.9 \%$ polymorphism and yielded 54 bands among 16 S. occidentalis samples and 35 of which were polymorphic with $64.8 \%$ polymorphism.

7 out of 85 SCoT primers could produce specific bands to distinguish S. obtusifolia from S. occidentalis. The primer SCoT21 produced a unique band about 2800 bp in S. occidentalis which could distinguish $S$. obtusifolia from S. occidentalis (Fig. 1). The other 6 SCoT primers also yielded different specific bands which could distinguish these two species (Additional file 1: Figure S1). 7 SCoT primers totally yielded 131 bands among $20 \mathrm{~S}$. obtusifolia samples, among which $112(85.5 \%)$ were polymorphic, and produced 92 bands among $16 \mathrm{~S}$. occidentalis samples, among which 78 $(84.8 \%)$ were polymorphic. 
Table 2 Genetic polymorphism parameters of 36 samples generated by 3 ISSR and 7 SCoT primers

\begin{tabular}{|c|c|c|c|c|c|c|c|}
\hline \multirow[t]{2}{*}{ Primer } & \multirow{2}{*}{$\begin{array}{l}\text { Primer sequence } \\
\left(5^{\prime} \rightarrow 3^{\prime}\right)\end{array}$} & \multicolumn{2}{|c|}{ Total bands } & \multicolumn{2}{|c|}{ Polymorphic bands } & \multicolumn{2}{|c|}{ PPB (\%) } \\
\hline & & Sob & Soc & Sob & Soc & Sob & Soc \\
\hline UBC880 & $(G A) 8 Y G$ & 29 & 21 & 20 & 15 & 68.9 & 71.4 \\
\hline UBC881 & GGGT(GGGGT)2G & 23 & 17 & 17 & 12 & 73.9 & 70.6 \\
\hline UBC885 & $\mathrm{BDB}(\mathrm{CA}) 7$ & 18 & 16 & 14 & 8 & 77.8 & 50.0 \\
\hline Average & - & 23.3 & 18.0 & 17.0 & 11.7 & 73.5 & 64.0 \\
\hline Total & - & 70 & 54 & 51 & 35 & 72.9 & 64.8 \\
\hline SCOT 8 & ACCATGGCTACCACCGCA & 20 & 15 & 18 & 12 & 90.0 & 80.0 \\
\hline SCOT 21 & CCATGGCTACCACCGCCT & 24 & 17 & 20 & 14 & 83.3 & 82.4 \\
\hline SCOT 27 & ACAATGGCTACCACTGCC & 16 & 11 & 15 & 10 & 93.8 & 90.9 \\
\hline SCoT 42 & ACCATGGCTACCACCGGC & 18 & 11 & 14 & 10 & 77.8 & 90.9 \\
\hline SCOT 43 & ACCATGGCTACCACCGCC & 15 & 12 & 11 & 11 & 73.3 & 91.7 \\
\hline SCOT 44 & ACGACATGGCGACCCACA & 17 & 13 & 16 & 11 & 94.1 & 84.6 \\
\hline SCoT 66 & ACAATGGCTACCACTAGC & 21 & 13 & 18 & 10 & 85.7 & 76.9 \\
\hline Average & - & 18.7 & 13.1 & 16.0 & 11.1 & 85.4 & 85.7 \\
\hline Total & - & 131 & 92 & 112 & 78 & 85.5 & 84.8 \\
\hline
\end{tabular}

Sob: S. obtusifolia; Soc: S. occidentalis; PPB: percentage of polymorphic bands

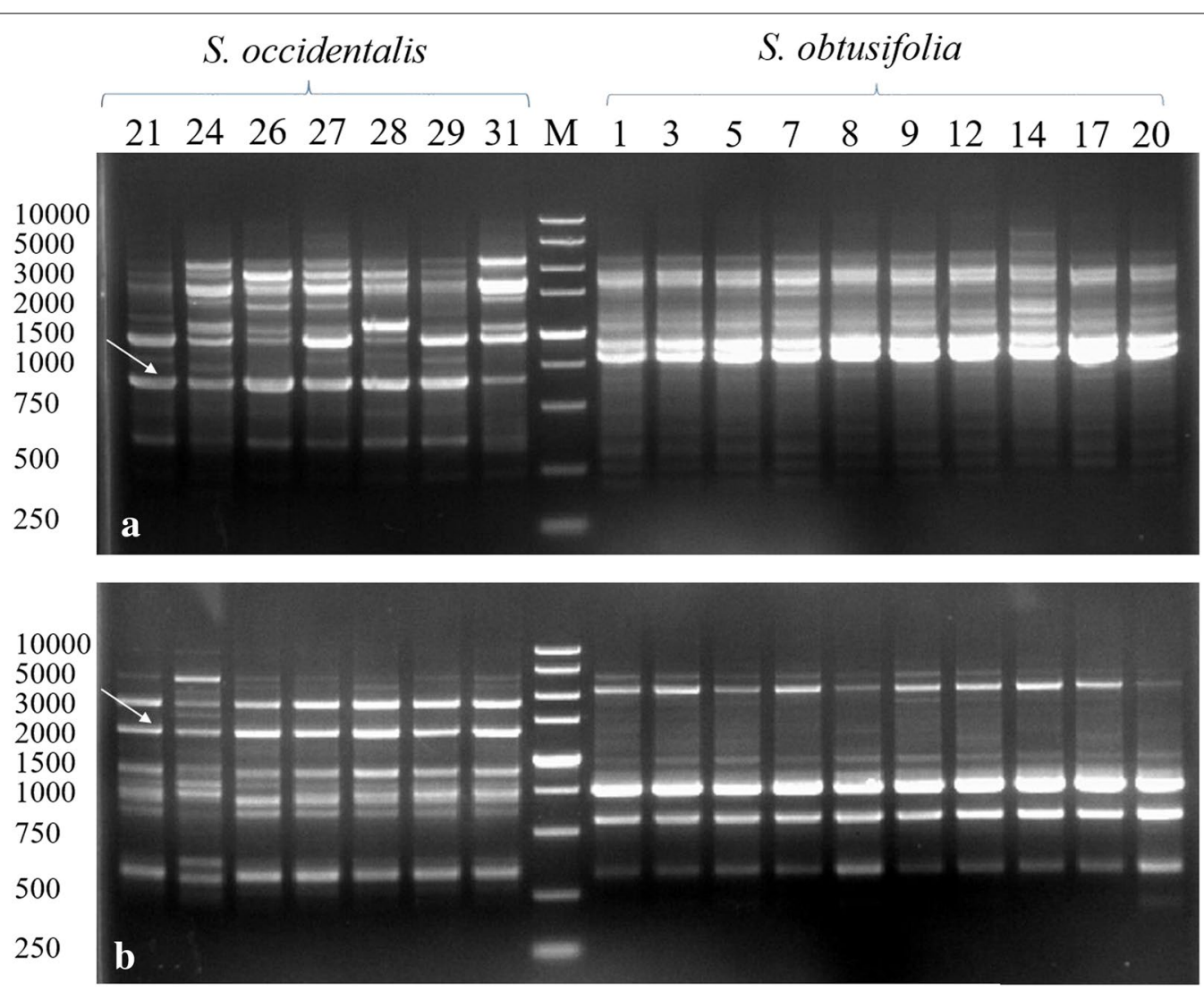

Fig. 1 The specific band (arrow indicate) generated by primers UBC80 (a) and SCOT 21 (b). The top row numbers were same with the samples number in Table 1.7 S. occidentalis samples and 10 S. obtusifolia samples were seperated by DL5000 Marker (TaKaRa) 

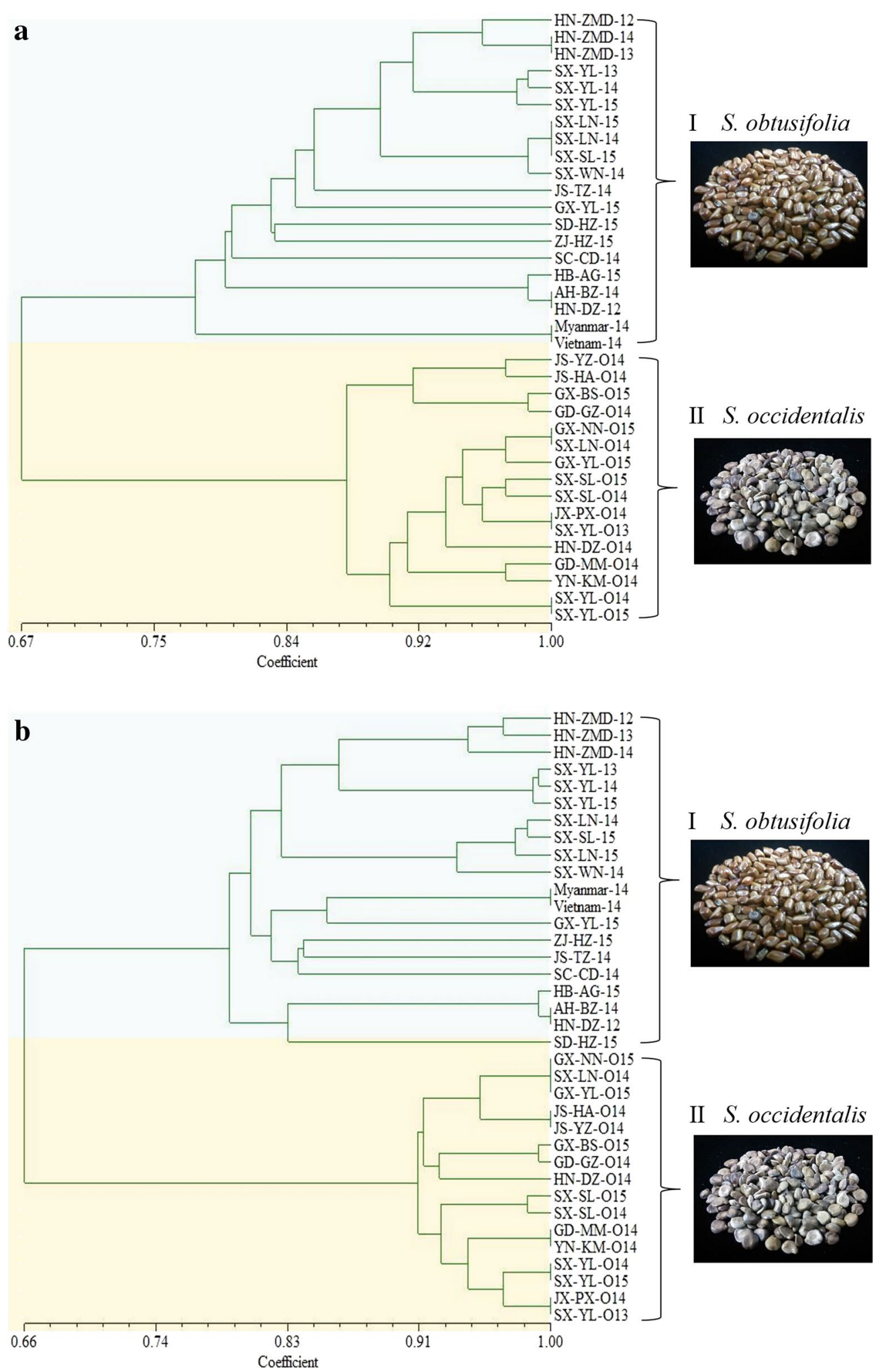

Fig. 2 UPGMA dendrograms generated based on ISSR (a) and SCoT (b). The cluster I contained 20 S. obtusifolia samples and cluster II contained 16 S. occidentalis samples, which indicated in blue and pink respectively. The photographs of S. obtusifolia and S. occidentalis were also exhibited 


\section{Clustering analysis}

The UPGMA dendrogram generated by ISSR divided 36 samples into two clusters (Fig. 2a). 20 S. obtusifolia samples and $16 \mathrm{~S}$. occidentalis samples were assigned into cluster I and cluster II, respectively. Most samples were clustered based on their geographic origin. For example, in cluster I, the samples collected from SX-YL and HN-ZMD during 2012-2015 years were clustered together, respectively, and other four samples from Shaanxi province were nested firstly. The samples from different regions did not show a clear cluster pattern. Two samples of Myanmar-14 and Vietnam-14 were clustered and separated from samples of China. Cluster II contained all S. occidentalis samples. SX-LN-O14 was clustered with GX-NN-O15, and SX-YL-O13 was clustered with JX-PX-O14. The remaining samples were clustered based on their geographic regions.

The UPGMA dendrogram generated by SCoT also assigned S. obtusifolia samples and S. occidentalis samples to two different clusters (Fig. 2b). In cluster I, the samples from Shaanxi and Henan showed same cluster pattern with ISSR dendrogram. While the samples of Myanmar-14 and Vietnam-14 were mixed with China samples which were different from ISSR dendrogram. In cluster II, SX-LN-O14 and JX-PX-O14 were grouped with two samples from Guangxi and three samples from Shaanxi, respectively, which was not consist with their origin.

\section{Sequences variations and genetic distance of intra- and inter-specific}

ITS2 sequences of 20 S. obtusifolia samples and 16 S. occidentalis samples were 233 bp. 20 S. obtusifolia samples represented 3 haplotypes namely, A1 (included 8 samples, see Table 1), A2 (2 samples) and A3 (10 samples). All 36 ITS2 of S. obtusifolia and S. occidentalis samples were submitted to GenBank and gained the accession numbers (Table 1). A1 showed the same ITS2 sequences with Senna obtusifolia voucher PS1588MT08 (GenBank: GQ434816). A2 and A3 showed the same sequence with voucher PH16960 (KX675057) and PH01988 (KX674796) respectively. Compared to A1, A2 showed a variation at $201 \mathrm{bp}$ with 'A' to 'G' and A3 showed a variation at $216 \mathrm{bp}$ with 'A' to 'C'. ITS2 sequences of 20 S. obtusifolia samples exhibited $99.79 \%$ similarity (Additional file 2: Figure S2). Compared with the existence S. occidentalis ITS2 sequence (JQ301840), no variations were detected among 16 S. occidentalis samples (haplotypes B1). Nevertheless, compared to the high similarity value of intra-specific, the inter-specific similarity value of $S$. obtusifolia and $S$. occidentalis was $89.58 \%$ (Additional file 2: Figure S2).

The genetic distance of $S$. obtusifolia ranged from 0 to 0.01 with an average of 0.003 . This value of $S$. occidentalis was 0 . However, the genetic distance between $S$. obtusifolia and S. occidentalis ranged from 0.280 to 0.290 with the average of 0.286 . The smallest genetic distance was detected between S. obtusifolia and S. tora (0.06), while the largest genetic distance was observed between $S$. obtusifolia and Glycine max (0.80). The results showed that the intra-specific genetic distances $(0.003)$ was lower than the inter-specific genetic distances $(0.286)$ between S. obtusifolia and S. occidentalis. The largest intra-specific distance was smaller than the distance of inter-specific, which could use as a parameter to distinguish S. obtusifolia from S. occidentalis.

\section{Phylogenetic analysis}

Two phylogenetic trees were constructed using Neighbor Jointing (NJ) and Maximum likelihood (ML) methods, respectively. Two phylogenetic trees showed large similarity with slightly difference (Fig. 3). 15 closely related species samples selected from GenBank were inserted into phylogenetic trees. Two S. tora were grouped with S. obtusifolia samples in both phylogenetic trees. Another difference was the branch consisted of 3 Senna spectibilis samples (KY492297-99) appeared a close evolution relationship with S. obtusifolia in NJ-tree (Fig. 3a), while it showed a relative far relationship with $S$. obtusifolia in ML-tree (Fig. 3b). Senna uniflora (KY492305) and Senna italic (JQ301844) exhibited the different positions in two trees. Despite showing some differences, both phylogenetic trees assigned $S$. obtusifolia samples and $S$. occidentalis samples to different branches (Fig. 3). Being the outgroup, three Glycine max samples were clustered together and separated from Senna samples. Collectively, the constructed phylogenetic trees could differentiate $S$. obtusifolia from S. occidentalis and elucidate the evolution relationship of Senna species.

\section{Determination of secondary metabolites}

Representative chromatograms of each five samples of S. obtusifolia and S. occidentalis were shown in Fig. 4. The concentrations of five secondary metabolites of each sample had been listed in Additional file 3: Table S1. The HPLC fingerprints of two species showed some similarities. At the retention times of 29.33, 30.42, 33.11 and $35.23 \mathrm{~min}$, these two species showed the same peaks. However, the peak detected at 12.95 min which was identified as aurantio-obtusin, was observed in S. obtusifolia samples only (Fig. 4). The concentrations of five secondary metabolites exhibited a large variation among different samples. The concentration of aurantio-obtusin and chrysophanol among S. obtusifolia samples ranged from 0.058 to $0.231 \%$ and 0.182 to $0.641 \%$, respectively (Additional file 3: Table S1). Chrysophanol concentration of $S$. occidentalis seeds ranged from 0.125 to $0.315 \%$. Physcion concentration ranged from 0.021 to 0.972 and 0.021 to $0.582 \%$ for S. obtusifolia and S. occidentalis, respectively. 


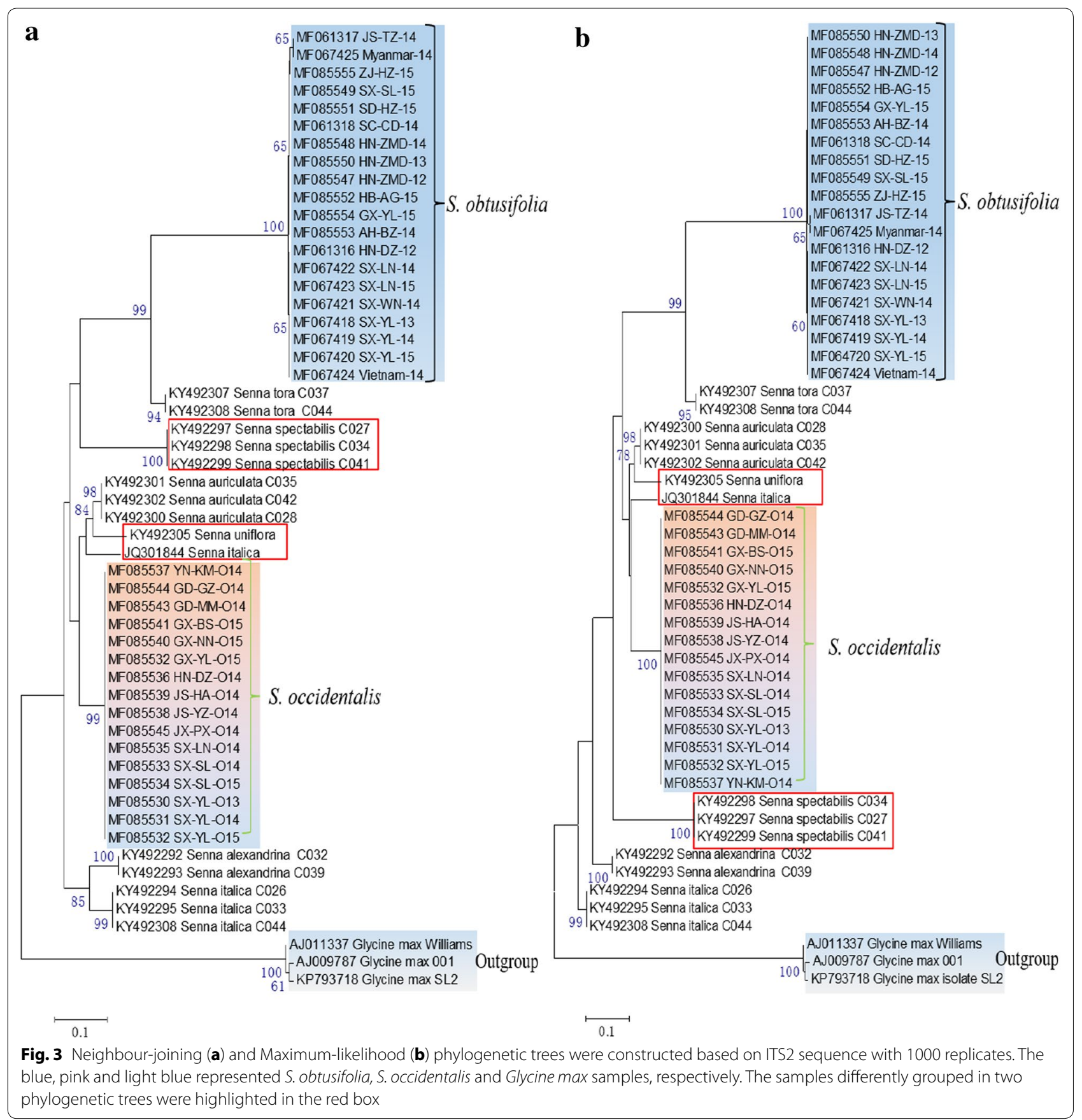

\section{Discussion}

Traditional appearance identification offered a direct method to identify medicinal herbs, but this method easily made confusion. It was more difficult to distinguish the real medicinal herb from its adulterants when they were adulterated. In addition, using one identification method only may not be suitable or convenient for all users. Molecular markers, ITS2 sequence and HPLC fingerprint were employed to evaluate the similarities and differences between S. obtusifolia seeds and S. occidentalis seeds, which could provide a suitable method to identify these two valuable medicinal herbs effectively.

Molecular markers had been widely used to identify the different genotypes of the species or the different ecological types with the same genotype. Several successful studies of plant identification were reported using different molecular marker systems. Based on selected RAPD, ISSR and SSR markers, Jatropha curcas samples 


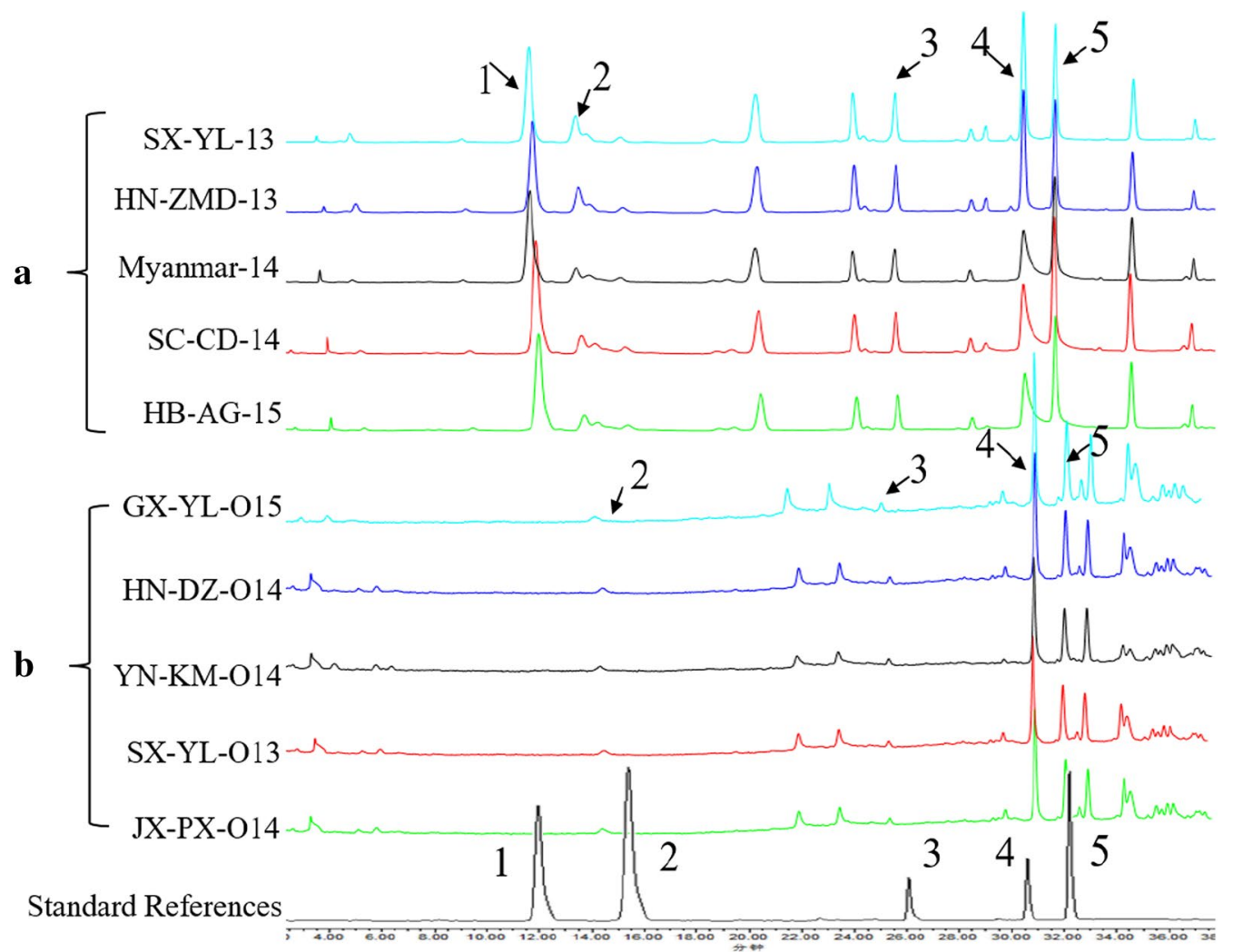

Fig. 4 HPLC fingerprints of five S. obtusifolia (a) and S. occidentalis samples (b). Standard references from 1 to 5 represented aurantio-obtusin, emodin, aloe-emodin, chrysophanol and physcion, respectively

from Mexica showed clear distinction with samples from other countries (Pamidimarri et al. 2009). Validation of the primers on Jatropha curcas indicated specificity of 3 RAPD primers to toxic genotypes, 5 RAPD and 2 ISSR primers to non-toxic samples and 4 RAPD primers to Mexican samples (Basha and Sujatha 2007). The study on Olea europaea indicated that 39 RAPD primers and 12 ISSR primers were able to distinguish 10 cultivars and 6 cultivars, respectively (Brake et al. 2014). In the present study, 3 ISSR primers and 7 SCoT primers could generate specific band to distinguish S. obtusifolia from S. occidentalis, which present a new method for identifying $S$. obtusifolia. Additionally, the results of molecular markers exhibited that both S. obtusifolia and S. occidentalis had a high level of genetic diversity and their genetic relationship showed a certain connection with geographical origin. This phenomenon could be explained by the wide cultivation area of S. obtusifolia and S. occidentalis in China for their medicinal and viewing purposes, which prompted their genetic diversity level at the same time.

The medicinal herbs collected from different regions showed large difference in quality. The adulterants exist is another reason accounting for the unstable quality of medicinal herbs. Identification of medicinal herbs based on morphological characters, microstructure or other traditional methods are lacking of the uniform standard and reliable data, which impede the further use of the medicinal herbs. Chen et al. (2010) proposed that ITS2 is a suitable DNA barcoding for medicinal plants. In recent years, ITS2 is a newly rising method which could effectively solve morphological indistinguishable or complex classification event. Additionally, ITS2 played a pivotal role in promoting the identification authenticity and stability of the medicinal herbs. ITS2 had been successively applied in identifying Hyoscyamus niger (Xiong et al. 2016) and Officinal rhubarb (Zhou et al. 2017). In this study, 36 samples of S. obtusifolia and S. occidentalis were collected from different geographic regions and evaluated their differences by ITS2. The sequence similarity of intra-specific was 99.79 and $100.0 \%$ for S. obtusifolia and S. occidentalis accordingly, while the similarity of inter-specific was $89.58 \%$. This results demonstrated that ITS2 was a suitable DNA barcoding for identification of S. obtusifolia. NJ-tree separated S. obtusifolia and S. occidentalis into different branches despite that they had a close relationship. Three Glycine max samples formed 
the outgroup and separated from Senna species. To further evaluate the genetic relationship of Senna species and offer a more accurate evolution view, ML-tree was also constructed. Showing slight difference with NJ-tree, ML-tree also assigned S. obtusifolia and S. occidentalis to different branches based on ITS2 sequence. It is no doubt that using two methods to construct phylogenetic trees is helpful in ensuring the accuracy of the results and could provide more information.

Containing different kinds of secondary metabolites was a significant feature to distinguish medicinal herbs from crops or other plants. The concentration of secondary metabolite was also an indispensable parameter to evaluate the quality of the medicinal herbs. The secondary metabolite determination by HPLC can be used as a powerful tool to identify the authenticity of medicinal herbs (Tian et al. 2009; Zhang et al. 2014). In this study, the HPLC fingerprints of S. obtusifolia and S. occidentalis showed some similarities since they both belong to the genus Senna family leguminous. The Chinese pharmacopoeia identified Juemingzi as the dried and ripe seeds of S. obtusifolia or S. tora, not included S. occidentalis seeds. As a famous traditional Chinese medicine, there had already been some HPLC fingerprint reports on S. obtusifolia seeds (Wang et al. 2009; Huang et al. 2010). However, to our knowledge, no HPLC fingerprint research was carried out on S. occidentalis seeds. In this study, we presented the HPLC fingerprint of S. occidentalis seeds for the first time. Notably, aurantio-obtusin was not detected in S. occidentalis samples, which was a significant difference from S. obtusifolia. Former researches focus on chemical constituents also indicated that S. occidentalis seeds did not contain aurantio-obtusin (Yadav et al. 2010). Same to our expectation, the concentration of chrysophanol and several other anthraquinones, i.e., emodin, aloe-emodin and physcion in S. obtusifolia seeds were higher than that in S. occidentalis seeds. This results implied that $S$. occidentalis seeds maybe not suitable for medicinal use.

There is no doubt that every method has its advantages and deficiencies. Molecular marker is easy to operate and does not need expensive experimental equipment, but the number of available primers is limited. ITS2 has high accuracy and low identification error rate. However, this method requires expensive DNA sequencer and needs to further analyze after obtaining the raw sequences data, which restrict the use of this method more or less. HPLC method is affordable, accurate and could simultaneously determine the concentration of various metabolites in medicinal materials. However, the processing of the sample preparation is cumbersome and the instrument operation has a certain degree of difficulty. In this study, we presented three methods to distinguish S. obtusifolia from S. occidentalis, aiming to provide a suitable method for users from different industrials to distinguish these two species effectively.

\section{Conclusions}

In summary, three approaches employed in this study could effectively identify S. obtusifolia seeds and S. occidentalis seeds. Selected 3 ISSR and 7 SCoT primers distinguished S. obtusifolia from S. occidentalis based on UPGMA dendrogram and agarose gel electrophoresis. ITS2 results showed that the intra-specific similarity of S. obtusifolia and S. occidentalis were 99.79 and $100.0 \%$, respectively. While the inter-specific similarity between S. obtusifolia and S. occidentalis was $89.58 \%$. Phylogenetic analysis assigned S. obtusifolia and S. occidentalis to different branches. HPLC fingerprints showed that two species shared some secondary metabolites, but S. occidentalis seeds did not contain aurantio-obtusin which could differentiate S. obtusifolia from S. occidentalis.

\section{Additional files}

Additional file 1: Figure S1. The specific bands (arrow indicate) generated by ISSR and SCOT primers. The first lane numbers were same with the sample number in Table 1.7 S. occidentalis samples and 10 S. obtusifolia samples were separated by DL5000 Marker (TaKaRa).

Additional file 2: Figure S2. Multiple sequences blast of ITS2 sequence of 20 S. obtusifolia samples (A) and 16 S. occidentalis samples (B) showed the intra-specific similarity was $99.79 \%$ and $100.0 \%$, respectively. Interspecific similarity value (C) of S. obtusifolia and S. occidentalis was $89.58 \%$.

Additional file 3: Table S1. Concentrations (\%) of five secondary metabolites of 20 S. obsusifolia samples and 16 S. occidentalis samples (Means $\pm S D, n=3$ ).

\section{Abbreviations}

ISSR: inter-simple sequence repeat; SCOT: target start codon polymorphism; ITS2: internal transcribed sequence 2; Sob: Senna obtusifolia; Soc: Senna occidentalis; PPB: percentage of polymorphic bands; HPLC: high performance liquid chromatography.

\section{Authors' contributions}

MRJ and LZS conceived the research; MRJ, XPG, ZHG and LFH collected the samples. MRJ and HZG performed the experimental work; MRJ, HZG, LY and HRL analyzed the data; MRJ, XPG and LZS wrote the manuscript. All authors read and approved the final manuscript.

\section{Author details}

1 State and Local Joint Research Center of TCM Fingerprint and NP Library, College of Life Sciences, Northwest A\&F University, Yangling 712100, Shaanxi, China. ${ }^{2}$ College of Life Sciences, Zhejiang Sci-Tech University, Hangzhou 310018, Zhejiang, China. ${ }^{3}$ Tianjin Tasly Modern TCM Resources Co. Ltd., Tianjin 300400, China. ${ }^{4}$ Shaanxi Tasly Plants Pharmaceutical Co. Ltd., Shangluo 726000, Shaanxi, China.

\section{Acknowledgements}

We thank Sheng Yang and Fuping Wang (Shaanxi Tasly Plants Pharmaceutical Co. Ltd.) for their help in collecting samples.

\section{Competing interests}

The authors declare that they have no competing interests. 
Availability of data and materials

Not applicable.

\section{Consent for publication}

Not applicable.

\section{Ethics approval and consent to participate}

Not applicable.

\section{Funding}

This work was financially supported by China National Twelfth "Five Year" Science and Technology Support Project (2015BAC011303) and the Major Industrial Cluster Project of Shaanxi Province (2012KTCL02-07).

\section{Publisher's Note}

Springer Nature remains neutral with regard to jurisdictional claims in published maps and institutional affiliations.

Received: 26 July 2017 Accepted: 2 October 2017

Published online: 02 November 2017

\section{References}

Basha SD, Sujatha M (2007) Inter and intra-population variability of Jatropha curcas (L.) characterized by RAPD and ISSR markers and development of population-specific SCAR markers. Euphytica 156:375-386

Brake M, Migdadi H, Al-Gharaibeh M, Ayoub S, Haddad N, Oqlah AE (2014) Characterization of Jordanian olive cultivars (Olea europaea L.) using RAPD and ISSR molecular markers. Hortic Sci 176:282-289

Chen SL, Yao H, Han JP, Liu C, Song JY, Shi LC, Zhu YJ, Ma XY, Gao T, Pang XH, Luo K, Li Y, Li XW, Jia XC, Lin YL, Leon CJ (2010) Validation of the ITS2 region as a novel DNA barcode for identifying medicinal plant species. PLOS ONE 5:e8613

Chinese Pharmacopoeia Commission (2015) Pharmacopoeia of People's Republic of China, Part 1. Chemical Industry Press, China, p 145 (In Chinese)

Collard BCY, Mackill DJ (2009) Start codon targeted (SCOT) polymorphism: a simple, novel DNA marker technique for generating gene-targeted markers in plants. Plant Mol Biol Rep 27:86-93

Drever BD, Anderson WGL, Riedel G, Kim DH, Ryu JH, Choi D, Platt B (2008) The seed extract of Cassia obtusifolia offers neuroprotection to mouse hippocampal cultures. J Pharmacol Sci 107:380-392

Huang XP, Zhong GY, Zhang XM (2010) Determination of aurantio-obtusin and chrysophanol in Cassiae Semen by HPLC. Chin J Chin Mater Med 35:2065-2067 (In Chinese)

Jafri MA, Subhani MJ, Javed K, Singh S (1999) Hepatoprotective activity of leaves of Cassia occidentalis against paracetamol and ethyl alcohol intoxication in rats. J Ethnopharmacol 66:355-361

Ju MS, Kim HG, Choi JG, Ryu JH, Hur J, Kim YJ, Oh MS (2010) Cassiae semen, a seed of Cassia obtusifolia, has neuroprotective effects in Parkinson's disease models. Food Chem Toxicol 48:2037-2044

Kim DH, Kim S, Jung WY, Park SJ, Park DH, Kim JM, Cheong JH, Ryu JH (2009) The neuroprotective effects of the seeds of Cassia obtusifolia on transient cerebral global ischemia in mice. Food Chem Toxicol 47:1473-1479

Li XE, Guo BL (2002) Effect of protein and anthraquinone glucosides from cassia seed on serum lipid of hyperlipidemia rats. Chin J Chin Mater Med 5:374-376 (In Chinese)

Li S, Di Y, Wang Y, Tan C, Fang X, Zhang Y, Zheng Y, Li L, He H, Li S, Hao X (2010) Anthraquinones and lignans from Cassia occidentalis. Helv Chim Acta 93:1795-1802

Pamidimarri DV, Singh S, Mastan SG, Patel J, Reddy MP (2009) Molecular characterization and identification of markers for toxic and non-toxic varieties of Jatropha curcas L. using RAPD, AFLP and SSR markers. Mol Biol Rep 36:1357-1364

Panigrahi G, Tiwari S, Ansari KM, Chaturvedi RK, Khanna VK, Chaudhari BP, Vashistha VM, Raisuddin S, Das M (2014) Association between children death and consumption of Cassia occidentalis seeds: clinical and experimental investigations. Food Chem Toxicol 67:236-248
Rohlf FJ (1993) NTSYS.PC. Numerical taxonomy and multivariate analysis system. Version 2.11Q. Applied biostatistics Inc, New York

Saganuwan AS, Gulumbe ML (2006) Evaluation of in vitro antimicrobial activities and phytochemical constituents of Cassia occidentalis. Animal Res Int 3:566-569

Sob SVT, Wabo HK, Tane P, Ngadjui BT, Ma D (2008) A xanthone and a polyketide derivative from the leaves of Cassia obtusifolia (Leguminosae). Tetraheron 64:7999-8002

Tamura K, Stecher G, Peterson D, Filipski A, Kumar S (2013) MEGA6: molecular evolutionary genetics analysis version 6.0. Mol Biol Evol 30:2725-2729

Teles AV, Fock RA, Gorniak SL (2015) Effects of long-term administration of Senna occidentalis seeds on the hematopoietic tissue of rats. Toxicon 108:73-79

Tian R, Xie P, Liu H (2009) Evaluation of traditional Chinese herbal medicine: Chaihu (Bupleuri Radix) by both high-performance liquid chromatographic and high-performance thin-layer chromatographic fingerprint and chemometric analysis. J Chromatogr A 1216:2150-2155

Tona L, Cimanga RK, Mesia K, Musuamba CT, De Bruyne T, Apers S, Hernans N, Miert SV, Pieters L, Totté J, Vlietinck AJ (2004) In vitro antiplasmodial activity of extracts and fractions from seven medicinal plants used in the Democratic Republic of Congo. J Ethnopharmacol 93:27-32

Wang WY, Zhao Q, Zhang TJ, Zhu HJ, Li Y (2009) HPLC fingerprint and chemical pattern recognition of Semen Cassiae. Chin Tradit Herbal Drugs 10:1638-1641 (In Chinese)

Xiong C, Hu ZG, Tu Y, Liu HG, Wang P, Zhao MM, Shi YH, Wu L, Sun W, Chen SL (2016) ITS2 barcoding DNA region combined with high resolution melting (HRM) analysis of Hyoscyami Semen, the mature seed of Hyoscyamus niger. Chin J Nat Med 14:898-903

Yadav JP, Arya V, Yadav S, Panghal M, Kumar S, Dhankhar S (2010) Cassia occidentalis L.: a review on its ethnobotany, phytochemical and pharmacological profile. Fitoterapia 81:223-230

Yang J, Ye H, Lai H, Li S, He S, Zhong S, Chen L, Peng A (2012) Separation of anthraquinone compounds from the seed of Cassia obtusifolia L. using recycling counter-current chromatography. J Sep Sci 35:256-262

Zhang R, Chen J, Shi Q, Li Z, Peng Z, Zheng L, Wang X (2014) Quality control method for commercially available wild Jujube leaf tea based on HPLC characteristic fingerprint analysis of flavonoid compounds. J Sep Sci $37: 45-52$

Zhou Y, Du X, Zheng X, Huang M, Li Y, Wang X (2017) ITS2 barcode for identifying the officinal rhubarb source plants from its adulterants. Biochem Syst Ecol 70:177-185

\section{Submit your manuscript to a SpringerOpen ${ }^{\circ}$ journal and benefit from:}

- Convenient online submission

- Rigorous peer review

- Open access: articles freely available online

- High visibility within the field

- Retaining the copyright to your article

Submit your next manuscript at springeropen.com 